\title{
Technical Guidelines for COVID-19 Laboratory Testing
}

\author{
Chinese Center for Disease Control and Prevention
}

These technical guidelines are formulated to guide disease control agencies and relevant institutions at all levels to carry out laboratory testing for 2019 novel coronavirus (2019-nCoV).

\section{SPECIMEN COLLECTION}

\section{Collection Targets}

Collection targets include the following: suspected cases, clustered cases, and other cases requiring diagnosis or differential diagnosis for 2019-nCoV; or other environmental or biological substances that require further screening and testing.

\section{Collection Requirements}

1. The 2019-nCoV testing specimens shall be collected by qualified technicians who have received biosafety training (who have passed the training) and are equipped with the corresponding laboratory skills. Personal protective equipment (PPE) requirements for sampling personnel are: N95 masks or masks with higher filtration efficiency, goggles, protective clothing, double-layer latex gloves and waterproof boot covers; if exposed to patients' blood, body fluids, secretions or excretions, the outer layer of the latex gloves should be changed in time.

2. Specimens of inpatient cases shall be collected by medical staff of the hospital where they are being treated.

3. Specimens of close contacts shall be collected by the designated local CDCs and medical institutions.

4. Multiple specimens may be collected in the course of the disease, depending on the need for laboratory testing.

\section{Categories of Specimens Collected}

Respiratory tract specimens in the acute phase (including upper or lower respiratory tract specimens) must be collected from each case; lower respiratory tract specimens shall be preferred for the collection from severe cases. Stool samples, whole blood samples, serum samples urine specimens can be collected according to clinical needs.

Categories of specimen:

Upper respiratory tract specimens: including nasopharyngeal swabs, pharyngeal swabs, etc.

Lower respiratory tract specimens: including deepcough sputum, alveolar lavage fluids, bronchial lavage fluid, and respiratory tract extracts.

Fecal specimens/anal swabs: Fecal samples are about $10 \mathrm{~g}$ (peanut size). If it is not convenient to collect fecal samples, an anal swab can be collected.

Blood specimens: One should, as much as possible, collect anticoagulated blood in the acute phase within 7 days after the onset of the disease. A $5 \mathrm{~mL}$ quantity of blood is required for each collection. Vacuum tubes containing EDTA anticoagulant are recommended in blood collection.

Serum specimens: Both acute-phase and convalescent serum specimens should be collected as often as possible. The first serum specimen should be collected as soon as possible (preferably within 7 days after the onset of illness), and the second specimen should be collected during 3 or 4 weeks after the onset of illness. A $5 \mathrm{~mL}$ quantity of blood is required for each specimen and vacuum tubes without anticoagulant are recommended. Serum specimens are mainly used for measuring antibodies, rather than nucleic acid testing.

Urine specimens: Collect $2-3 \mathrm{~mL}$ mid-stream of morning urine sample.

\section{Methods of Specimen Collection and Processing}

Nasopharyngeal swab: The sampler gently holds the person's head with one hand, the swab in another, insert the swab via nostril to enter, slowly get deep along the bottom of the lower nasal canal. Because the nasal canal is curved, do not force too hard to avoid traumatic bleeding. When the tip of the swab reaches the posterior wall of the nasopharyngeal cavity, rotate gently once (pause for a moment in case of reflex cough), then slowly remove the swab and dip the swab tip into a tube containing $2-3 \mathrm{~mL}$ virus preservation solution (or isotonic saline solution, tissue culture solution, or phosphate buffer), discard the tail, and tighten the cap.

Pharyngeal swab: the sampled person first gargles with normal saline, the sampler then immerses the swabs in sterile saline (virus preservation solution is not allowed to avoid antibiotic allergies), holds the head of the sampled person up slightly with his or her mouth 
wide open making an "ah" sound to expose the lateral pharyngeal tonsils, insert the swabs, stick across the tongue roots, wipe both sides of the pharyngeal tonsils with pressure at least 3 times, then wipe on the upper and lower walls of the pharynx for at least 3 times, dip the swabs in a tube containing $2-3 \mathrm{~mL}$ storage solution (or isotonic saline solution, tissue culture solution, or phosphate buffer solution), discard the tail, and tighten the cap. The pharyngeal swabs can also be placed in the same tube together with the nasopharyngeal swab.

Nasopharyngeal or respiratory tract extract: Extract mucus from the nasopharynx or extract respiratory secretions from the trachea with a collector connected to a negative-pressure pump; insert the head of the collector into the nasal cavity or trachea, turn on the negative pressure, rotate and slowly withdraw the head of the collector, collect the extracted mucus, and rinse the collector once with $3 \mathrm{~mL}$ of the sampling solution (a pediatric catheter connected to a $50 \mathrm{~mL}$ syringe may be used as an alternative to the collector).

Deep cough sputum: Ask the sampled person to cough deeply and collect the sputum coughed up in a screw-capped plastic tube containing $3 \mathrm{~mL}$ of the sampling solution. If the sputum is not collected in the sampling solution, $2-3 \mathrm{~mL}$ of the sampling solution can be added into the tube before testing, or sputum digestion reagents (Table 1) of equal volume to the sputum can be added.

TABLE 1. Formula of storage fluid for sputum digestion reagents.

\begin{tabular}{lc}
\hline \multicolumn{1}{c}{ Ingredients } & Mass/volume \\
\hline Dithiothreitol & $0.1 \mathrm{~g}$ \\
Sodium chloride & $0.78 \mathrm{~g}$ \\
Phosphorus chloride & $0.02 \mathrm{~g}$ \\
Disodium hydrogen phosphate & $0.112 \mathrm{~g}$ \\
Potassium dihydrogen phosphate & $0.02 \mathrm{~g}$ \\
Water & $7.5 \mathrm{~mL}$ \\
$\mathrm{pH} 7.4 \pm 0.2\left(25^{\circ} \mathrm{C}\right)$ & \\
\hline
\end{tabular}

Dilute the storage solution to $50 \mathrm{~mL}$ with deionized water before use. Sputum can also be liquefied with a phosphate buffer containing $1 \mathrm{~g} / \mathrm{L}$ of protease $\mathrm{K}$ in an equal volume of sputum.

Bronchial lavage fluid: Insert the head of the collector into the trachea (about $30 \mathrm{~cm}$ deep) from the nostril or the tracheal insertion part, inject $5 \mathrm{~mL}$ of physiological saline, turn on the negative pressure, rotate the head of the collector and slowly withdraw it. Collect the extracted mucus and rinse the collector once with the sampling solution (a pediatric catheter connected to a $50 \mathrm{~mL}$ syringe may be used as an alternative to the collector). The sampling method depends on the hospital.

Alveolar lavage fluid: After local anesthesia, insert a bronchoscope through the mouth or nose, pass through the pharynx into the branch of the right middle lobe or the lingular segment of the left lung, and insert the tip into the bronchial branch opening; slowly add sterilized physiological saline through the biopsy hole of the bronchoscope, with $30-50 \mathrm{~mL}$ of saline each time, $100-250 \mathrm{~mL}$ in total, $300 \mathrm{~mL}$ at most. The sampling method depends on the hospital.

Fecal specimen: Take $1 \mathrm{ml}$ of the sample processing solution (Table 2), pick up a little sample about the size of a soybean and add it into the tube, gently blow for 3-5 times, set aside at room temperature for 10 minutes, centrifuge at $8,000 \mathrm{rpm}$ for 5 minutes, and absorb the supernatant for detection.

TABLE 2. Formula of fecal sample processing solution.

\begin{tabular}{lc}
\hline \multicolumn{1}{c}{ Ingredients } & Mass/volume \\
\hline Tris & $1.211 \mathrm{~g}$ \\
Sodium chloride & $8.5 \mathrm{~g}$ \\
$\begin{array}{l}\text { calcium chloride anhydrous or calcium } \\
\text { chloride containing crystalline water }\end{array}$ & $1.1 \mathrm{~g} \mathrm{or} 1.47 \mathrm{~g}$ \\
$\begin{array}{l}\text { Deionized Water } \\
\text { Adjusted pH to } 7.5 \text { with concentrated hydrochloric acid and } \\
\text { replenishing with deionized water to } 1,000 \mathrm{~mL} .\end{array}$ \\
\hline
\end{tabular}

Fecal sample processing solution can be prepared inhouse by the laboratory.

Stool suspensions can also be prepared using HANK's solution or other isotonic saline solution, tissue culture solution, or phosphate buffer solution. If the patient has symptoms of diarrhea, collect 3-5 mL of stool specimen, gently blow and mix, centrifuge it at $8,000 \mathrm{rpm}$ for 5 minutes, and absorb the supernatant to reserve for use.

Anal swab: Gently insert the disinfectant cotton swab into the anus for $3-5 \mathrm{~cm}$ in depth, then gently rotate and pull out, immediately put the swab into a screw-capped sampling tube containing 3-5 mL virus preservation solution, discard the tail, and tighten the tube cover.

Blood samples: It is recommended to use vacuum blood vessels containing EDTA anticoagulant to collect $5 \mathrm{~mL}$ of blood samples. Nucleic acid extraction should be performed on whole blood or plasma according to the type of nucleic acid extraction reagent selected. For plasma separation, the whole blood should be centrifuged at 1,500 to $2,000 \mathrm{rpm}$ for 10 minutes, and the supernatant should be collected in a sterile plastic tube with a screw cap. 
Serum specimen: Collect a $5 \mathrm{~mL}$ blood specimen with a vacuum negative-pressure blood collection tube. Keep the specimen at room temperature for 30 minutes, centrifuge it at 1,500-2,000 rpm for 10 minutes, and collect the serum in a sterile plastic tube with a screw cap.

Other materials: To be collected in a standardized manner in accordance with design requirements.

\section{Specimen Packaging}

Collected specimens shall be packaged separately in a biosafety cabinet of a BSL-2 laboratory.

1. All specimens should be placed in an airtight freeze-tolerant sample collection tube of appropriate size with a screw cap and a gasket inside. The sample number, category, name, and sampling date should be indicated on the outside of the container.

2. Specimens kept in an airtight container should be sealed in a plastic bag of appropriate size, with each bag containing one specimen. The specimen packaging requirements must meet the corresponding standards of the Technical Regulations for the Safe Transport of Dangerous Goods by Air.

3. Prior to transportation, external specimens shall undergo the three-layer packaging applicable to Category A and Category B infectious substances based on the categories of the specimens.

\section{Specimen Preservation}

Specimens for virus isolation and nucleic acid detection purposes should be tested as soon as possible. Specimens to be tested within 24 hours can be stored at $4{ }^{\circ} \mathrm{C}$; those that cannot be tested within 24 hours should be stored at $-70{ }^{\circ} \mathrm{C}$ or below (specimens may be temporarily stored in $-20{ }^{\circ} \mathrm{C}$ refrigerators in the absence of $-70{ }^{\circ} \mathrm{C}$ storage condition). Serum can be stored at $4{ }^{\circ} \mathrm{C}$ for 3 days and below $-20{ }^{\circ} \mathrm{C}$ for a longer period. A special depot or cabinet is required to store specimens separately. Repeated freeze-thaw cycles during specimen transportation should be avoided.

\section{Specimen Submission and Examination}

Collected specimens should be sent to laboratories as soon as possible. Dry ice and other refrigeration methods are recommended for the preservation of specimens to be transported over long distances.

\section{Submission of specimens}

Specimens of cluster cases in each provincial-level administrative division shall be submitted to the National Institute for Viral Disease Control and Prevention (NIVDC) of China CDC for testing and review with the specimen submission form attached
(seeSupplementary Figure S1, available in http://weekly. chinacdc.cn/).

\section{Pathogen and specimen transportation}

1. Domestic transport

Strains of 2019-nCoV or other potentially infectious biological substances are subject to packaging instructions for Category A substances assigned to UN2814, and the PI 602 of the Technical Instructions For The Safe Transport of Dangerous Goods by Air (Doc 9284) issued by ICAO; environmental samples, assigned to UN3373, shall be transported in Category B packaging in accordance with the PI 650, Doc 9284; one may refer to the aforementioned standards for specimens to be transported in other modes of transportation.

A Permit of Transport is required for the transportation of the 2019-nCoV strains or other potentially infectious substances according to the Transport Regulations on the Highly Pathogenic Microorganism (Virus) Strains and Specimens that are Pathogenic to Humans (Order No. 45, former Ministry of Health).

2. International transport

Standard packaging shall be applied to 2019-nCoV strains or samples to be transported internationally, with relevant procedures handled in accordance with the Provisions on the Administration of the Health Quarantine of Entry/Exit Special Articles as well as relevant national and international requirements.

3. Management of strains and samples

2019-nCoV strains and samples should be managed by designated personnel with accurate records of the source, category, quantity, and registration number of the strains and samples. Effective measures should be adopted to ensure the security of the strains and samples. Efforts should be made to prevent the misuse, malicious use, theft, robbery, loss, and leakage of the strains and samples.

\section{LABORATORY TESTING OF THE 2019-NCOV}

\section{Real-Time Fluorescence-based Reverse \\ Transcriptase Polymerase Chain Reaction (RT-PCR) assay of the 2019-nCoV Nucleic Acid \\ 2019-nCoV nucleic acid assay}

The nucleic acid detection method introduced in this guideline mainly targets at open reading frame $1 \mathrm{ab}$ 
(ORF lab) and nucleocapsid protein $(\mathrm{N})$ genes in the 2019-nCoV genome.

Target 1 (ORF 1ab):

Forward primer (F): CCCTGTGGGTTTTACACT TAA

Reverse primer (R): ACGATTGTGCATCAGCTGA Fluorescent probe (P): 5' -FAM-CCGTCTGCGGT ATGTGGAAAGGTTATGG-BHQ1-3'

Target $2(\mathrm{~N})$ :

Forward primer (F): GGGGAACTTCTCCTGCTA GAAT

Reverse primer (R): CAGACATTTTGCTCTCAA GCTG

Fluorescent probe (P): 5' -FAM-TTGCTGCTGCTT GACAGATT-TAMRA-3'

For nucleic acid extraction and real-time fluorescence-based RT-PCR reaction system and reaction conditions, refer to kit instructions of the manufacturers concerned.

\section{Judgment of results}

Negative: no Ct value or $\mathrm{Ct}$ value is 40 .

Positive: Ct value $<37$.

Gray zone: Repeated experiments are recommended if $\mathrm{Ct}$ values range between 37 and 40 . If the $\mathrm{Ct}$ value reads $<40$ and the amplification curve has an obvious peak, the sample should be considered to be positive, otherwise it should be considered as negative.

Note: If a commercial kit is used, the instructions provided by the manufacturer will take priority.

\section{Confirmation of cases}

Laboratory confirmation of positive cases requires one of the following two conditions:

1. The real-time fluorescence-based RT-PCR assay of $2019-\mathrm{nCoV}$ in the same specimen shows that the two targets, ORF1ab and Protein N, are both positive. In case of the result showing positive for one target, then samples shall be re-collected for another test. If it is still positive for a single target, the result should be deemed positive.

2. The real-time fluorescence-based RT-PCR assay of two types of specimens show one single target as positive at the same time, or one target as positive in two samples of the same type, the result should be deemed positive.

Negative nucleic acid results cannot rule out 2019$\mathrm{nCoV}$ infections. Factors leading to false negatives shall be precluded including: poor quality of samples, for instance the respiratory tract samples of the oropharynx and other parts; samples collected too early or too late; samples that are improperly stored, transported, or processed; technical reasons such as virus mutations, PCR inhibition, etc.

\section{Serum Antibody Tests}

Serum antibody tests (colloidal gold, magnetic particle chemiluminescence, ELISA) are used as supplementary tests for cases of negative 2019-nCoV nucleic acid tests, used in conjunction with nucleic acid tests in the diagnosis of suspected cases, or used in serological surveys and past exposure surveys of concerned population groups. Laboratory confirmed positive cases need to meet one of the following two conditions:

1. Serum IgM antibodies and/or IgG antibodies to 2019-nCov are positive;

2. Serum IgG antibodies to 2019-nCov turn from negative to positive or the $\mathrm{IgG}$ antibody titers of recovery period are 4 times or more higher than that of acute phase.

Using serum in the acute phase within 7 days after the onset of disease detects IgM and IgG, if the test result is negative, repeat collection for testing within 10 days after the onset of disease is recommended. Convalescent serum specimens within 3-4 weeks after the onset of illness should be used for detecting IgG. Instructions from the manufacturer's manual should be followed for commercial testing kits.

\section{BIOSAFETY REQUIREMENTS FOR PATHOGEN EXPERIMENTS}

According to the biological features, epidemiological characteristics, clinical data, and other available information concerning the 2019-nCoV, the pathogen shall be temporally managed as a Category $\mathrm{B}$ pathogen and microorganism based on its hazards. Specific requirements are listed as follows:

\section{Viral Culture}

Viral culture refers to operations such as virus isolation, culture, titration, neutralization test, purification of live virus and its protein, lyophilization of virus, and recombination test to produce live virus. The above operations should be performed in a biosafety cabinet in a BSL-3 laboratory. When viral medium is used to extract nucleic acid, the addition of lysing agent or inactivating agent must be performed under the same level of laboratory and protective conditions as viral culture. Laboratories shall report to the National Health Commission for approval and obtain relevant qualifications before carrying out the corresponding activities. 


\section{Animal Infection Experiments}

Animal infection experiments refer to operations such as infecting animals with live viruses, sampling of infected animals, processing and testing of infectious samples, special test for infected animals, disposal of infected animal excrement, etc., which should be performed in a biosafety cabinet in a BSL-3 laboratory. Laboratories shall report to the National Health Commission for approval and obtain relevant qualifications before carrying out the corresponding activities.

\section{Operations of Uncultured Infectious Substances}

Operations of uncultured infectious substances refer to viral antigen detection, serological testing, nucleic acid extraction, biochemical analysis, inactivation of clinical samples, and other operations performed on uncultured infectious substances before inactivation through a reliable method. The operation should be performed in a BSL-2 laboratory with personal protective equipment subject to BSL-3 laboratory protection requirements.

\section{Operations of Inactivated Substances}

After reliable inactivation of infectious substances or live viruses, operations such as nucleic acid testing, antigen testing, serological testing, and biochemical analysis should be performed in a BSL-2 laboratory. Molecular cloning and other operations not involving live pathogenic viruses may be carried out in a BSL-1 laboratory.

Acknowlegement: China CDC Weekly would like to thank Xijuan Fu of the WHO China Office, Xiaoping Dong, Ning Feng, Wenling Wang, and Weiyi Li of China CDC, Guogao Wu (WPRO/WHO retiree), and Guyu Li (an American licensed physician) for their contributions in translating and editing this document (1).

This Chinese document written by experts in China CDC Laboratory Detection Technology Group for COVID-19 Emergency Response, including Wenling Wang, Roujian Lu, Peihua Niu, Li Zhao, Huijuan Wang, Guizhen Wu, and Wenjie Tan of National Institute for Viral Disease Control and Prevention, China CDC.

doi: $10.46234 / \mathrm{ccdcw} 2020.085$

Submitted: March 10, 2020; Accepted: March 12, 2020

\section{REFERENCES}

\footnotetext{
1. Chinese Center for Disease Control and Prevention. Technical documents for COVID-19 prevention and control. http://www.chinacdc. cn/jkzt/crb/zl/szkb_11803/jszl_11815/202003/t20200309_214241.html. [2020-3-9]. (In Chinese)
} 


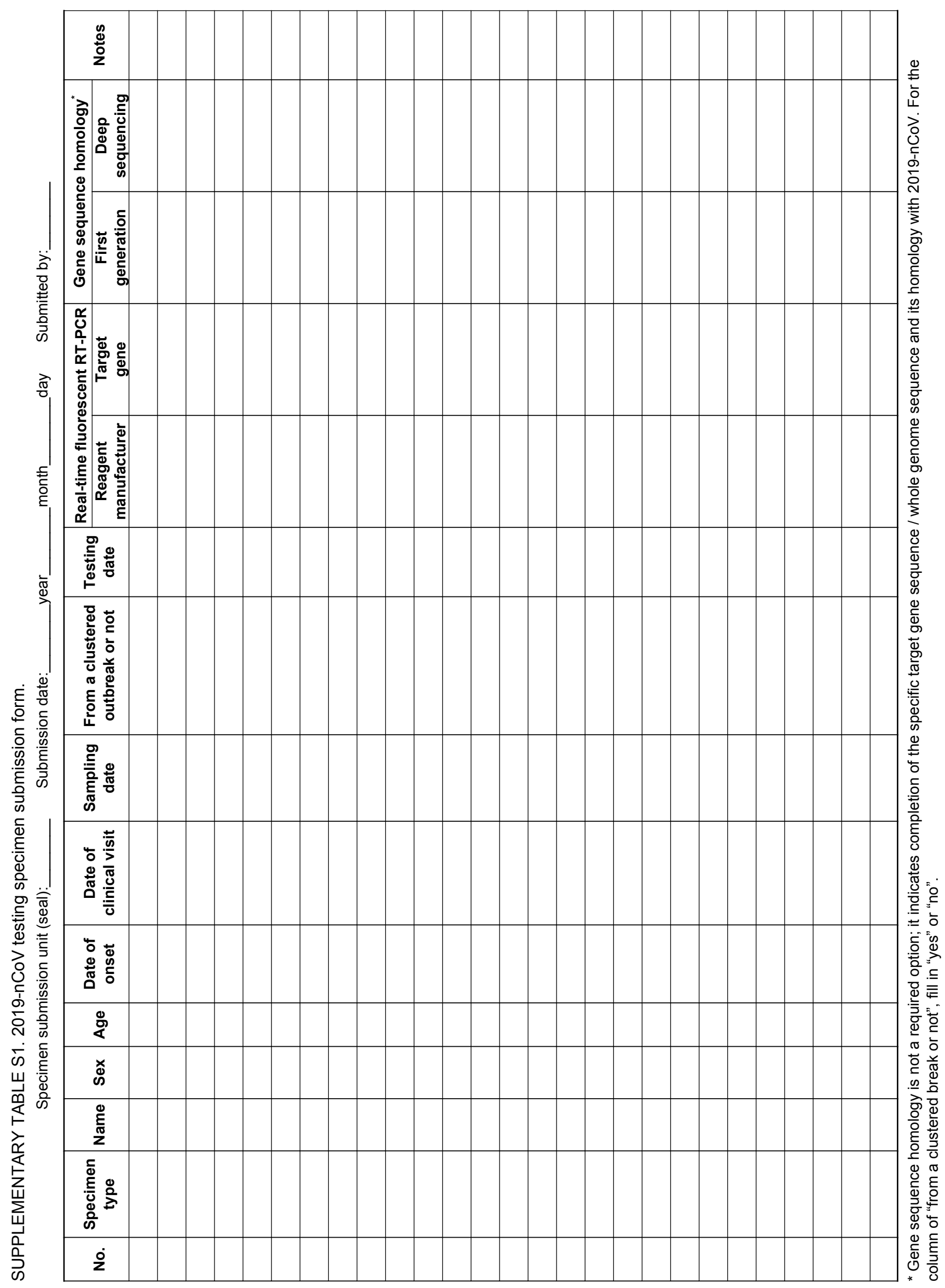

